\title{
Cambio de perspectiva en los paradigmas y prácticas profesionales en el acompañamiento de personas que usan sustancias psicoactivas: análisis desde la Psicología y la Terapia Ocupacional.
}

Change of perspective in paradigms and professional practices in the accompaniment of people who use psychoactive substances: Analysis from Psychology and Occupational Therapy

Filiación:

${ }^{1}$ Sistema de Estudios de Postgrado. Maestría profesional en el Estudio de las Adicciones, Universidad Estatal a Distancia UNED, San José, Costa Rica. ${ }^{2}$ Instituto sobre Alcoholismo y Farmacodependencia - IAFA, San José, Costa Rica y Escuela de Terapia Ocupacional, Universidad Santa Paula, San José, Costa Rica

Correspondencia: $\square$ Javier Vindas Acosta. Email: jvinacost@ gmail.com

Financiamiento:

Ninguno

Conflictos de Interés: Ninguno

Forma de citar: Vindas-Acosta J, Valdelomar-Marín E. Cambio de perspectiva en los paradigmas y prácticas profesionales en el acompañamiento de personas que usan sustancias psicoactivas: Análisis desde la Psicología y la Terapia Ocupacional. Rev Ter. 2021;15(1): 119-133

Abreviaturas: OMS:

Organización Mundial de la Salud; OPS: Organización Panamericana de la Salud; SPA: Sustancia

Psicoactiva.

Fecha de recepción: 10 de noviembre de 2020.

Fecha de aceptación: 6 de enero del 2021.
Javier Vindas-Acosta $\square$ 1, Erick Valdelomar-Marín ,2

\section{Resumen}

La siguiente es una revisión conceptual gestionada a partir de la experiencia desarrollada en Costa Rica sobre el abordaje de personas que usan sustancias psicoactivas, desde diferentes paradigmas de atención de salud que han atravesado la forma en que se entiende, acompaña, atiende e interviene los trastornos de sustancias psicoactivas y se ofertan modalidades de servicios gestionados por el estado y la sociedad civil. Para esto partimos de la premisa que el consumo de sustancias psicoactivas son un hecho histórico-cultural-socioeconómico, en la relación ser humano y el medio ambiente en el desarrollo de la humanidad. El objetivo es brindar una reflexión que permita a los lectores un cuestionamiento sobre sus creencias y la relación de estas con las prácticas de prestación de servicios, para identificar desde qué paradigma se están brindando las mismas. Como conclusiones de los autores, se propone que aun hoy paradigmas históricos (desde miradas mecanicistas, centradas en la enfermedad, con un enfoque bio-médico predominante y la radicalidad de la abstinencia como única meta posible), que ya parecían haber sido superados e integrados, atraviesan el entendimiento y acompañamiento de profesionales y servicios que atienden personas que usan sustancias psicoactivas: lo que limita una implementación de estrategias e intervenciones integrales con enfoque de derechos humanos, desarrolladas en co-construcción con las personas, familias y comunidades de acuerdo a su realidad y necesidades.

Palabras clave: trastornos relacionados con sustancias, terapia ocupacional, psicología

\section{Abstract}

The following is a conceptual review managed from the experience developed in Costa Rica on the approach of people who use psychoactive substances from different health care paradigms that have gone through how it is understood, accompanied, attended, intervened, and offered modalities of services managed by the state and civil society. 
For this, we start from the premise that the consumption of psychoactive substances is a historical-culturalsocioeconomic fact, in the relationship between human beings and the environment in the development of humanity. The objective is to provide a reflection that allows readers to question their beliefs and their relationship with service provision practices, to identify from which paradigm they are being provided. As the authors' conclusions, it is proposed that still historical paradigms (from mechanistic perspectives, focused on the disease, with a predominant bio-medical approach and the radically of abstinence as the only possible goal). That has already been overcome and integrated; go through the understanding and support of professionals and services that accompany people who use Psychoactive Substances, which limits the implementation of comprehensive intervention strategies with a human rights approach, developed in co-construction with individuals, families, and communities according to their reality and needs.

Key words: substance-related disorders, occupational therapy, psychology

\section{Introducción}

Para entender el uso de sustancias psicoactivas (SPA) en las personas, es necesario partir de una visión estructural del fenómeno, que tome en cuenta las diversas dimensiones que presenta este tópico, tales como los aspectos histórico-ideológicos, geopolíticos, biológicos, psicológicos, sociales, ocupacionales, espirituales, socio-familiares, teórico-metodológicos y los de tratamiento y seguimiento.

Las tendencias de avanzada apuntan en nuestros tiempos al enfoque centrado en la persona, familia y comunidad como corolario de las visiones ideológico-culturales anteriores, las cuales promueven una participación activa de las personas en sus procesos de cuidado de la salud ${ }^{1}$. Según la OMS $^{2}$ :

...es necesario garantizar la implementación, de manera multisectorial y coordinada, de un paquete de estrategias que abarque todo el proceso de salud pública, desde la prevención primaria y la reducción del riesgo hasta la gestión de los trastornos por consumo de drogas, rehabilitación, atención, que se base en los preceptos fundamentales de equidad, justicia social y derechos humanos, haciendo hincapié en los países y las poblaciones más necesitadas y prestar la debida consideración a los determinantes económicos, sociales y ambientales de la salud, la ciencia y la evidencia de intervenciones basadas en la gente y enfoques centrados en las personas

Esta participación activa de las personas en los procesos de salud ha sido de difícil manejo por parte de los equipos interdisciplinarios, siguiendo a De las Heras ${ }^{3}$ esto es debido en parte a convicciones preconcebidas relacionadas con modelos mecanicistas, a estigmas que sin pensarlo son fomentados por los mismos profesionales y a la falta de confianza de los profesionales con respecto a las habilidades y potenciales de la persona que presentan problemas de cualquier tipo. Compartimos este análisis de que los profesionales han tendido a rotular y centrarse en la disminución de los síntomas y conductas - actitudes catalogadas como "mal adaptadas", por no tener enfoques claros sobre los aspectos motivacionales-volitivos que implican los procesos de cambio y sobre la interacción dinámica entre factores personales, ambientales y estructurales. 
Este proceso perceptivo se reproduce también en los usuarios de SPA, cuando se auto estigmatizan y se sienten merecedores del rechazo social (culpa por el consumo o por la recaída, sentirse como un criminal o mantenerse en el anonimato social). El auto-estigma podría definirse como un proceso des adaptativo en el que la persona acepta prejuicios sociales e integra esta creencia como parte de su autoconcepto ${ }^{4}$. A este proceso de interiorización de estereotipos lo llamaremos aquí ideologización del uso de sustancias psicoactivas.

Esta revisión es una propuesta dialéctica entre premisas provenientes de la psicología y la ocupación humana, como posturas científicas liberadoras que potencian y cristalizan estrategias de atención centrada en la persona, familia, comunidades y contextos en situaciones sociales y de salud derivadas del uso de SPA, integrando y superando las perspectivas de las posturas tradicionales centradas en la abstinencia, la moralenfermedad y los aspectos jurídico-legales. La presente propuesta tiene como intención brindar miradas y herramientas que se complementen con las estrategias de mayor validez (entrevista motivacional, intervenciones breves, abordaje comunitario), que desarrollan profesionales, voluntarios, líderes y pares en diferentes dispositivos; así como la posibilidad de generar en el lector su propia reflexión sobre cuál es su postura ante el entendimiento del uso de SPA, que permea su estilo profesional en la prestación de servicios.

Desde lo anterior la visión filosófica general debe orientar las dimensiones ontológica (forma del objeto salud y su interpretación), epistemológica (formas de relación sujeto-objeto y su cosmovisión) y praxiológica (concepción de la práctica y posicionamiento frente a la estructura de poder).

\section{Discusión}

Para poder describir el cambio de paradigma en las estrategias de atención de profesionales y personal técnico, dirigidas a personas con trastornos por el uso de drogas ${ }^{5}$ en la última década en Costa Rica; iniciaremos con una revisión de la construcción social del fenómeno droga, continuaremos con un análisis filosófico, estructural y general, de modo que oriente las dimensiones ontológicas, epistemológica y praxiológica del quehacer analítico ${ }^{6}$.

\section{La construcción social del fenómeno droga}

La ideología es el conjunto de costumbres, hábitos y creencias que comparte una organización histórico social y que influye en la concepción del mundo de los individuos ${ }^{7}$. La concepción del mundo es lo que cada persona interpreta de esa ideología (lo individual que reproduzco) y el paradigma es una estructura de conocimientos filosófico-científicos que es compartida según cada momento histórico. Siguiendo a Kuhn ${ }^{8}$ tanto la ideología, como la concepción de mundo y los paradigmas (verdades aceptadas y compartidas), nos atraviesan a todos y condicionan a las personas, en las formas de pensar, actuar y relacionarse con el mundo, la sociedad y los otros ${ }^{9}$, generando una influencia directa sobre la concepción de lo que se considera ontológicamente el ser humano o la persona ${ }^{10}$.

El abordaje que se brinda a las personas que usan SPA, tiene que ver con la concepción del ser humano que tiene cada sujeto (profesional o persona usuaria). Estos elementos socioculturales participan en la construcción de la identidad, la personalidad y la memoria del sujeto, lo cual permea a la vez, las formas del ejercicio profesional y la manera en que la persona entiende su propio consumo. Todo lo anterior va a influir en la metodología, técnicas 
de abordaje y en el establecimiento de la relación terapéutica para el acompañamiento de personas que usan sustancias.

Según Kornblit et $\mathrm{al}^{10}$ "el entender el consumo de SPA resulta una temática compleja porque para analizarlo se deben tener en cuenta la multiplicidad de elementos como las diferentes representaciones sociales, prácticas individuales y colectivas, los sujetos, las sustancias, los contextos socioeconómicos, políticos, culturales e ideológicos". Consideramos que Costa Rica se encuentra en un momento histórico en el que se están ampliando los paradigmas en base a los cuales se crearon y diseñaron los servicios del sistema de salud y los de la sociedad civil dirigidos a las personas que usan SPA.

\section{a. Naturalizar}

Goffman $^{11}$ plantea el concepto de normativización o naturalización, según el cual la sociedad establece estereotipos (cómo debe ser determinada especie de individuo), que se alinean a expectativas de lo que se considera normal, de modo que cuando las personas mantienen atributos que los diferencian de los demás resultan menos valoradas o consideradas débiles. Este rechazo social se traduce en estigma, es decir el rechazo social y la discriminación a los que son diversos y por tanto anormales.

Se normativiza cuando se trivializa el consumo de drogas legales y se ven como "decadentes" a los consumidores de drogas ilegales. Esta normativización lleva a la legitimación de posturas esteriopantes hacia el consumo de SPA ilegales, equiparando las representaciones sociales relacionadas al narcotráfico hacia las personas que usan drogas, etiquetándolas como criminales. Al respecto, Moscovici, citado por Mora ${ }^{12}$, afirma que la sociedad condiciona y normaliza por medio de "representaciones sociales", las que define como los sistemas de valores, ideas y prácticas para orientarse en el mundo, que proporcionan códigos comunes para nombrar aspectos del mundo, la historia y la sociedad. Es decir, en cada época histórica hay algo aceptado como bueno o malo y esas representaciones sociales impactan el entendimiento del uso de SPA.

Según Althusser ${ }^{13}$ la sociedad normativiza a través de aparatos ideológicos del estado, la familia, la educación, los medios de comunicación y la religión. Estos discursos político-ideológicos condicionan los discursos científicos, que a su vez se nutren de las relaciones de poder sociales, lo que es aceptado socialmente va a ser siempre validado por los discursos científicos de cada época.

Los cambios históricos plantean que haya revoluciones científicas, $\mathrm{Kuhn}^{8}$ anota, que cuando el paradigma aceptado se agota, porque se acumulan respuestas insatisfactorias, o no da respuestas adecuadas a las circunstancias reales que tiene la humanidad, surgen nuevos paradigmas. Actualmente Costa Rica, en consonancia con las buenas prácticas globales, se mueve de paradigmas tradicionales hacia paradigmas centrados en la persona, abordaje comunitario y los Derechos Humanos ${ }^{14-19}$.

\section{b. Estereotipos sociales, prejuicio y estigmatización}

Para De las Heras ${ }^{3}$, el estigma se refiere a una preconcepción errónea acerca de un fenómeno o circunstancia que lleva a la discriminación, no nace espontáneamente, por el contrario, se desarrolla con el tiempo en relación con la cultura predominante que impregna a los grupos sociales de valores, creencias y posturas que llevan a determinadas formas de accionar ante estos fenómenos y realidades humanas, como lo es el entendimiento 
del fenómeno de las drogas.

Otro concepto relacionado es el de estereotipos sociales, siguiendo a Cersósimo Guzmán ${ }^{20}$ es el que alude a creencias referidas a grupos, que son compartidas por los miembros de una cultura, por las que se adjudica un conjunto de atributos a un determinado grupo social. Con respecto al uso de SPA, existen estereotipos positivos y negativos. Los estereotipos positivos se presentan en la publicidad de las drogas legales, a través de los anuncios, los cuales ofrecen imágenes arquetípicas de personas con cuerpos bellos, felices, contentos, alegres, exitosos, lo que genera expectativas positivas alrededor del consumo y el condicionamiento reforzador del mismo. Los estereotipos negativos, que, desde nuestro criterio, han permeado los modelos tradicionales de atención en salud, se pueden sintetizar en cinco: la diferenciación entre drogas legales e ilegales, la diferenciación entre droga buena- droga mala, centrarse en el concepto de enfermedad, considerar la abstinencia como único indicador de éxito terapéutico y considerar la recaída como un fracaso (tabla1).

En cuanto al primer estereotipo, la diferenciación entre drogas legales e ilegales, las investigaciones nacionales y mundiales ${ }^{21-24}$ indican que las drogas legales son las que están relacionadas con mayores problemas en la salud pública. Sin embargo, se hace más énfasis en las propiedades decadentes de las drogas ilegales desde juicios morales y jurídico-legales, sin priorizar indicadores de salud de la población. Como señalan Amador et $\mathrm{al}^{25}$ :

El problema principal es que este enfoque no diferencia entre las sustancias que se consumen y los tipos de consumo, estereotipando a toda persona usuaria como un toxicómano o farmacodependiente. De hecho, en el mismo artículo 79 de la Ley 8204, se puede apreciar esta concepción que equipara el uso de drogas con la adicción y se promueve el tratamiento como único método para abordarla.

La influencia de esta falsa percepción condiciona la búsqueda tardía de acompañamiento por parte de personas que usan SPAlegales (alcohol/ tabaco/medicamentos) y presiona socialmente a las personas que usan SPA ilegales a recibir tratamiento.

El segundo estereotipo (la diferenciación entre droga buena- droga mala) hace énfasis en lo moral, cuando el criterio científico es el de que ninguna droga es inocua (inofensiva). Desde lo moral siempre va a haber un binomio ineludible entre pecado-expiación de culpa. Cuando se habla del "flagelo" de la droga, se establece una relación entre, por un lado, el castigo divino, la droga como castigo por una sociedad pecadora, y por otro se habilita una respuesta igualmente agresiva: al flagelo hay que "combatirlo" con medidas drásticas que depuren a la sociedad de este "mal"10. Hacer una diferencia entre drogas buenas y malas impide una posición científica-neutral que permita centrarse en la salud (por ejemplo, valorar a una persona que consume cannabis desde su individualidad y definir cuáles efectos tiene esta sustancia psicoactiva en su memoria, su comportamiento psicomotriz, en el síndrome amotivacional o cómo influencia su proyecto de vida). Las valoraciones morales extrapolan los estigmas que se relacionan con el narcotráfico hacia las personas que usan SPA ilegales, clasificándolos como individuos de segunda categoría y al mismo tiempo brindan un status quo de aceptación social a los que usan SPA legales.

El tercer estereotipo es el de enfermedad primaria (centrarse en el concepto de enfermedad), 
la OMS define el consumo perjudicial y el síndrome de dependencia como un trastorno ${ }^{5}$, pero lo anterior lleva a la tendencia a homogeneizar a los usuarios (todos son iguales) o trampa del etiquetaje que es estigmatizante ${ }^{26}$. Este estereotipo de "enfermedad" se concentra en hacer énfasis en una personalidad adicta (egocentrismo, negación, confrontación, coraza a quebrar) que refleja la trampa de confrontación-negación ${ }^{26}$. Además, hace énfasis en la pérdida de control y en la interpretación de que cualquier esfuerzo de control, es la manifestación patognomónica de la negación de la enfermedad. Contrario a este criterio de enfermedad Miller et $\mathrm{al}^{26}$ probaron que, para efectos del pronóstico no es necesario que una persona se reconozca como "enferma adicta"; lo que sí es necesario es que la persona reconozca que tiene un problema de control con el consumo. Este estereotipo plantea que la enfermedad es progresiva incurable y fatal ${ }^{27,28} \mathrm{y}$ se hace énfasis en la pérdida de control, es decir que las personas no pueden aprender a controlarse (consumir con un plan de moderación) de ninguna manera. Sin embargo, muchas personas con un consumo perjudicial o un síndrome de dependencia toman decisiones y logran generar estrategias de moderación ${ }^{27}$. Este estigma ejemplifica un reduccionismo biológico, entendiendo el uso de SPA exclusivamente como una enfermedad del cerebro, al respecto $\mathrm{Heim}^{29}$ refiere que el abuso de sustancias no es solo una consecuencia del mal funcionamiento cerebral, ya que no puede ser separado de sus contextos sociales, psicológicos, culturales, políticos, legales y ambientales.

Centrarse en el concepto de enfermedad genera la trampa del etiquetaje $e^{26,30}$, de persona recortada, inestable, efímera o la noción de que el usuario activo tiene conciencia y juicio deficiente, por lo que no puede participar significativamente en el plan de tratamiento ${ }^{27}$. Se duda de su competencia personal, lo que lleva a la Trampa del experto, la creencia de esta trampa es que, desde una relación vertical, no se confía en la capacidad de la persona para tener autonomía y capacidad de decisiones en sus vidas y cualquier esfuerzo por controlar se interpreta como negación ${ }^{27,28}$. Porque desde la posición del experto, el usuario tiene que reconocer primero su problema de dependencia para ser tratable, la realidad, en contraste con este estigma, nos dice que la gente resuelve lo que necesita resolver primero ${ }^{26,30}$. Este estereotipo dicta también que solamente a través de la confrontación se puede tratar a una persona con consumo problemático, esa es la trampa de confrontación-negación ${ }^{26,30}$.

Se cree que confrontando a la persona es como la persona va a entender su problema y no se comprende que lo que la confrontación hace es elevar defensas psicológicas de resistencia al cambio $^{27}$. A esto Tatarsky ${ }^{31}$ refiere que incluso se llega al punto que a los que quieren trabajar otros problemas se le niega la terapia. Encasillar afecta la individualidad. Cuando se le pide a una persona que acepte que es adicto, se le condiciona a verse y definirse únicamente desde una parte de sí mismo (la de consumidor) y se le impele a que minimice la importancia de otras facetas o fortalezas de su personalidad (como padre, como profesional, como deportista). Esto violenta los derechos de esa persona, porque le estamos diciendo que su adicción y su consumo definen su personalidad y su identidad. Primero se es persona que consumidor, siguiendo a Schnake ${ }^{32}$ el diagnóstico no se superpone a la persona.

El cuarto estereotipo es el de centrarse en la abstinencia total, como único indicador o criterio de éxito terapéutico. Aquí la visión de la recuperación es una visión lineal, ya que se cree idealmente 
que la recuperación tiene un inicio y un final. Esto es una trampa metodológica del terapeuta, que Miller et $a^{26,30}$ nombran como "la trampa del énfasis prematuro", que promulga que la acción terapéutica debe concentrarse en buscar la abstinencia inmediata. Contrario a esto Prochaska et $\mathrm{al}^{33}$, los cuales probaron que las personas cambian cuando ven más ganancia en el cambio, y se encuentran preparadas para el mismo. En relación al consumo de SPA, la abstinencia forzada es una variable importante en la recaída. A este respecto Prochaska et $\mathrm{al}^{33}$ prueban que solo el $5 \%$ de las personas consumidoras cambian sin retrocesos de modo que la abstinencia no es la única meta aceptable. De acuerdo con datos de IAFA podemos inferir que la mayoría de los usuarios mantienen moderación y la minoría son los que desarrollan dependencias ${ }^{21}$. Otra consecuencia de esta distorsión clínica siguiendo a Tatarsky ${ }^{31}$ es, que estar abstinente se asume como prerrequisito para el tratamiento y posterior seguimiento, lo que lleva a prácticas excluyentes y violatorias del Derecho Humano a la salud.

Otra consecuencia es que se cae en la trampa del énfasis prematuro ${ }^{26,30}$, esto es, por ejemplo, según Tatarsky ${ }^{31}$, cuando al ofertar el servicio de terapia lo que le pido primero a la persona es que detenga su consumo, como condición para darle ayuda, explica que el uso de SPA ha sido la única situación de salud donde se exige eliminar el síntoma para brindarle tratamiento. Nadie le pide a una persona con un cuadro clínico depresivo o ansioso que llegue al tratamiento sin la depresión o la ansiedad. La realidad en contraste, nos indica que hay personas con consumo problemático que logran moderación, u otros que logran reducir las consecuencias adversas de sus dependencias sin abstinencia. Con estas aseveraciones no se autoriza a la gente con una dependencia a seguir en su dependencia, tenemos que saber distinguir clínicamente las realidades individuales de las personas con las que trabajamos y saber que la responsabilidad terapéutica fundamental es darle a la persona las herramientas y recursos, para que pueda enfrentar y tomar decisiones en su relación con el uso de SPA. Si la persona decide seguir consumiendo, esa no debería de ser una razón para que se le excluya del tratamiento ${ }^{31}$.

El quinto estereotipo es ver la recaída como un fracaso. Los que recaen, son desvinculados de la mayoría de los programas ambulatorios y residenciales ${ }^{31,34-36}$, lo que lleva a señalamientos y juicios morales condenatorios de parte de los equipos de profesionales, familia y comunidad. Desde esta perspectiva la recaída es indicadora de que no hubo ningún progreso o acciones valiosas que quedan descalificadas por la recaída, lo cual tiene efecto de desmotivación en la persona que usa SPA. Cuando la persona se ve a sí misma desde esta visión moral recaída-fracaso, se genera lo que Marlatt et al ${ }^{35-36}$ Ilamó el Fenómeno Efecto de Violación de la Abstinencia o EVA, que produce culpa, vergüenza y desesperanza por atribuir la recaída a una debilidad interna en vez de una dificultad de aprendizaje para el manejo de los disparadores de ansiedad de consumo ${ }^{39,40}$. Los que recaen representan la mayoría, esta es una realidad clínica ${ }^{36}$. La recaída es una norma, no la excepción ${ }^{34,45}$.

\section{c. Enfoque de Reducción de Daños:}

En nuestro país, desde el año 2017 se aprueba el Modelo de Reducción de Daños para el abordaje del fenómeno de drogas, el cual es resultado de una política pública más participativa, que involucró a diferentes actores de sociedad civil y estado ${ }^{17}$. Esto ha permitido legitimar los recursos de base comunitaria, la diversificación de la oferta de servicios de atención del sistema nacional de 
tratamiento y la disminución de la brecha del acceso a la salud de los colectivos con mayor vulnerabilidad.

El principio terapéutico fundamental del enfoque de Reducción de Daños de acuerdo con Marlatt et $\mathrm{al}^{36}$, es que no podemos eliminar la respuesta adaptativa de la persona que consume mientras no tenga otros mecanismos de afrontamiento eficaces. En reducción de daños la abstinencia si es un objetivo, pero lo es cuando la persona que consulta lo plantee y no cuando se le dice que tiene que hacerlo ${ }^{31}$. Se reconoce el uso como un hecho, es por lo tanto una obligación bioética del terapeuta acompañarlo en ese proceso. Siguiendo a Tatarsky ${ }^{31}$, otros principios de reducción de daños son considerar a las personas como actores responsables de sus decisiones, apelando a la singularidad que distingue y hace única a cada persona. Con esto se busca evitar la etiqueta de patología, no estigmatizar, ya que se deben considerar tres elementos clínicos importantes cuando se valoran las condiciones de una persona usuaria de SPA: primero, el elemento de que el uso de sustancias varía en un continuo de uso ocasionalconsumo perjudicial-síndrome de dependencia según cada individuo; segundo que la motivación al cambio es fluctuante y tercero, que la persona consumidora mantiene en un alto porcentaje otros trastornos comórbidos, es decir, son heterogéneos. Tampoco se tiene una lectura centrada en los déficits, sino que, se basa en las habilidades, además del respeto por las fortalezas de la persona que consulta y su capacidad para cambiar. La relación terapéutica con la persona es horizontal, de modo que el plan individual de acompañamiento se diseña en coproducción terapeuta-persona consultante $26,30,31$.

Se trata de una filosofía pragmáticacompasiva, es decir, aplicar lo que sirve para reducir el daño y compasiva al relacionarse con respeto, dignidad e inclusión, desde un humanismo bioético que confía en la persona ${ }^{36}$. Miller et $\mathrm{al}^{27}$ proponen la sustitución del término "recaída" por el de "recurrencia" ya que el primero hace referencia a suposiciones binarias, sobre posibles resultados de éxito o fracaso. Según la OMS y la UNODC ${ }^{37}$ en los Estándares Internacionales del 2020, la recuperación es una serie de esfuerzos por aprender a vivir con una calidad de vida autorregulada, deteniendo o reduciendo el uso de sustancias, siempre con el fin de mejorar la salud, el bienestar y el funcionamiento social del individuo.

\section{Prácticas profesionales y desideologización para el acompañamiento de personas que usan SPA.}

Desde nuestra posición la atención en salud desideologizada, se comprende el fenómeno droga desde su arista del consumo, como una ocupación humana basada en sus características contextuales de producto social (determinantes sociales de la salud) y como una construcción social (estereotiposestigmas), basado en los aspectos filosóficopolíticos implicados, concentrándose en los efectos y consecuencias en la Salud Pública de dichos comportamientos, en especial de las poblaciones vulnerabilizadas.

Martín Baró ${ }^{38}$ plantea que asumir modelos transculturales y transhistóricos, elaborados en circunstancias distintas a las nuestras, puede llevarnos a una grave distorsión de lo que en realidad son nuestros pueblos y nuestras problemáticas. Por tanto, se debe reconocer la relación ideologizante entre la enajenación personal y enajenación social, que permita una conciencia que genere una nueva epistemología desde los saberes de los pueblos y de las poblaciones vulnerabilizadas, como lo son las personas que consumen SPA. 
Tabla 1. Estereotipos de uso de sustancias psicoactivas (SPA): del modelo tradicional hacia la Reducción de Riesgos y Daños en Costa Rica

\begin{tabular}{|c|c|c|c|c|}
\hline $\begin{array}{l}\text { Representación } \\
\text { social del } \\
\text { entendimiento }\end{array}$ & \multicolumn{2}{|c|}{ Construcción desde Paradigmas Tradicionales } & \multicolumn{2}{|r|}{$\begin{array}{c}\text { Construcción desde Reducción de Riesgos y Daños y Atención } \\
\text { Centrada en la Persona }\end{array}$} \\
\hline $\begin{array}{l}\text { 1-La } \\
\text { diferenciación } \\
\text { entre drogas } \\
\text { legales e } \\
\text { ilegales }\end{array}$ & $\begin{array}{l}\checkmark \\
\checkmark \\
\checkmark \\
\checkmark\end{array}$ & $\begin{array}{l}\text { Las SPA ilegales son más peligrosas que } \\
\text { las legales. } \\
\text { Los efectos dañinos de las SPA legales } \\
\text { se minimizan y los de las ilegales se ven } \\
\text { decadentes. } \\
\text { Influye en una respuesta retardada a } \\
\text { problemas de salud por uso SPA legales, } \\
\text { por el contrario, existe una mayor presión } \\
\text { de recibir tratamiento por uso de SPA } \\
\text { ilegales. } \\
\text { Percepción del usuario como desadaptado, } \\
\text { criminal. } \\
\text { Estilo profesional: Juzgador- Perseguidor }\end{array}$ & & $\begin{array}{l}\text { Ninguna Sustancia Psicoactiva es inocua-inofensiva } \\
\text { Los efectos de las drogas legales generan impacto significativo } \\
\text { en la salud pública. } \\
\text { Se debe responder primero a los problemas generados por } \\
\text { las SPA legales, ya que, epidemiológicamente, son las que } \\
\text { muestran mayor uso e impacto dañino en la salud pública; } \\
\text { incluyendo el uso de SPA ilegales con abordaje territorial. } \\
\text { Percepción del usuario como ser humano libre de escoger el } \\
\text { tipo relación que establece con la SPA. } \\
\text { Estilo profesional: Libre de juicios ético-morales }\end{array}$ \\
\hline $\begin{array}{l}\text { 2-La } \\
\text { diferenciación } \\
\text { entre droga } \\
\text { buena y droga } \\
\text { mala }\end{array}$ & $\begin{array}{l}\checkmark \\
\checkmark \\
\checkmark \\
\checkmark\end{array}$ & $\begin{array}{l}\text { Las SPA ilegales son un flagelo, las } \\
\text { legales naturalizadas como vehículos de } \\
\text { socialización recreativa. } \\
\text { Se demoniza y criminaliza el uso de } \\
\text { drogas. } \\
\text { Percepción del usuario como vicioso, } \\
\text { pecaminoso. } \\
\text { Estilo profesional: Salvador }\end{array}$ & $\checkmark$ & $\begin{array}{l}\text { Las SPA no son buenas ni malas, su peligrosidad depende } \\
\text { del significado, funcionalidad y forma de uso que le den las } \\
\text { personas, en un contexto socio-cultural- histórico. } \\
\text { El uso de sustancias es una ocupación humana no es ni buena } \\
\text { ni mala, sino una actividad con significado-intencionalidad para } \\
\text { la persona. } \\
\text { Persona que establece una relación con las SPA. } \\
\text { Estilo profesional: Acompañamiento respetuoso }\end{array}$ \\
\hline $\begin{array}{l}\text { 3- Centrarse en } \\
\text { el concepto de } \\
\text { enfermedad. }\end{array}$ & $\begin{array}{l}\checkmark \\
\checkmark \\
\checkmark \\
\checkmark\end{array}$ & $\begin{array}{l}\text { Todo uso lleva a la adicción (síndrome de } \\
\text { dependencia) } \\
\text { Hay personalidad adictiva con } \\
\text { egocentrismo, negación, manipulación, } \\
\text { pérdida de control. (Trampa del etiquetaje) } \\
\text { La adicción es una enfermedad del } \\
\text { cerebro, progresiva, incurable y fatal. } \\
\text { Estilo profesional: Relación vertical } \\
\text { entre el experto-paciente. Confrontación } \\
\text { de defensas (quebrarlo). (Trampa de } \\
\text { confrontación-negación y Trampa del } \\
\text { experto). }\end{array}$ & $\checkmark$ & $\begin{array}{l}\text { Existen diferentes formas de consumo, que varían desde, } \\
\text { el consumo ocasional, consumo perjudicial o síndrome de } \\
\text { dependencia. No hay una escalada lineal de una forma de } \\
\text { consumo a otra. } \\
\text { No existe personalidad adictiva, sino un problema de control } \\
\text { de las frecuencias e intensidades del consumo. La etiqueta } \\
\text { de adicto es estéril para el pronóstico. Primero se es persona } \\
\text { antes que consumidor. } \\
\text { El consumo problemático es una condición social y de salud } \\
\text { compleja que incluye aspectos políticos, culturales, legales, } \\
\text { psicológicos, ocupacionales, sociales y ambientales. } \\
\text { Estilo profesional: Acompañar a la persona a resolver su } \\
\text { ambivalencia, relación horizontal }\end{array}$ \\
\hline $\begin{array}{l}\text { 4- Considerar } \\
\text { la abstinencia } \\
\text { como único } \\
\text { indicador de } \\
\text { éxito terapéutico }\end{array}$ & $\begin{array}{l}\checkmark \\
\checkmark \\
\checkmark\end{array}$ & $\begin{array}{l}\text { Abstinencia como único objetivo } \\
\text { terapéutico aceptable y válido. } \\
\text { Recuperación desde visión lineal, con } \\
\text { inicio y final } \\
\text { Abstinencia como requisito para acceder a } \\
\text { tratamiento y mantener seguimiento } \\
\text { Estilo profesional: Rígido y condicionante. } \\
\text { (Trampa del énfasis prematuro) }\end{array}$ & $\checkmark$ & $\begin{array}{l}\text { La abstinencia es una alternativa entre diversidad de opciones } \\
\text { funcionales. } \\
\text { Recuperación desde la visión en espiral, entendida como un } \\
\text { proceso de aprendizaje con progresos-retrocesos. } \\
\text { No se exige abstinencia para brindar acompañamiento o excluir } \\
\text { de tratamiento. } \\
\text { Estilo profesional: parte de las necesidades, intereses y } \\
\text { realidades de la persona. }\end{array}$ \\
\hline $\begin{array}{l}\text { 5- Considerar la } \\
\text { recaída como un } \\
\text { fracaso. }\end{array}$ & $\checkmark$ & $\begin{array}{l}\text { Recaer es un fracaso que anula los } \\
\text { progresos logrados. } \\
\text { Se excluye a los usuarios de los procesos } \\
\text { de tratamiento y seguimiento. } \\
\text { Genera auto atribución de culpa, } \\
\text { vergüenza, autocastigo y desesperanza. } \\
\text { Estilo profesional: Rígido y condicionante, } \\
\text { señalador. Enfatiza la ineficacia personal. }\end{array}$ & $\checkmark$ & $\begin{array}{l}\text { Recaída es la norma no la excepción, dentro del proceso de } \\
\text { vida. } \\
\text { Inclusión de la recaída como parte de los procesos de } \\
\text { acompañamiento. } \\
\text { Reatribución de significado de recaída como una dificultad de } \\
\text { manejo del control de los riesgos y daños. } \\
\text { Estilo profesional: Centrado en la persona. Enfatiza la } \\
\text { autoeficacia y es desideologizado. }\end{array}$ \\
\hline
\end{tabular}


Dentro de las tareas de la Psicología de la Liberación, Martín Baró38-39 plantea la desideologización del sentido común y de la experiencia cotidiana, partiendo de que el conocimiento es una construcción social y este se pone al servicio de la ideología oficial cuando someten a los grupos sociales a la mentira de un discurso dominante, que niega, ignora o disfraza aspectos esenciales de la realidad, al naturalizar las desigualdades y estigmas, al aceptarlos como normales; como lo hemos ejemplificado anteriormente, al señalar las representaciones sociales y estereotipos hegemónicos en el entendimiento del fenómeno droga, que se replican en las prácticas profesionales colonizadas de América Latina.

Ante los sesgos mencionados, seguimos lo que marca Martín Baró respecto a tener una visión objetiva, al indicar sobre las SPA que "tan absurdos nos parecen los ataques basados en su nocividad fisiológica como las defensas incondicionadas que afirman su inocuidad"39. La desideologización apunta a la relación que establecen los individuos con las SPA en su contexto natural y de su impacto en la salud integral y bienestar, más que a valoraciones morales, jurídicas u orgánicas reduccionistas, superadas en un análisis integrador que parte como señala De Las Heras y otros del "hacer con los individuos" ${ }^{40}$, no desde los objetivos predefinidos de los proveedores de atención de salud.

Una actitud desideologizante es entender las realidades y necesidades de las poblaciones vulnerabilizadas históricamente, a esto Martín Baró $^{39}$ señala que la sociedad es generadora de consumidores, medidos según el valor en el mercado de la producción, donde las desigualdades generan trauma psicosocial. En esa dirección Cortés Amador et $\mathrm{al}^{41}$ indican "las personas y las poblaciones que tienen mayores riesgos de sufrir una violación a sus derechos humanos, son las que viven en mayores condiciones de vulnerabilidad social como por ejemplo personas usuarias de drogas, campesinos agricultores, vendedores de drogas al menudeo, "mulas" o "correos humanos".

Segúnnuestrapropuesta, elacompañamiento terapéutico desideologizado, debe abarcar los procesos de estigmatización y exclusión social de las personas que usan SPA generados sistémicamente y replicados en nuestra sociedad, familia y comunidades. Además, no parte de que la persona tiene que ser normal, sino de que la persona acepte su diferencia, su diversidad ${ }^{11}$. En ese sentido el acompañamiento terapéutico debe abordar la carga simbólica del estigma, es decir, intervenimos en la auto aceptación, autoestima, valoración personal y respeto por sí mismo y por las demás personas, y como lo indica Barrero Cuellar ${ }^{42}$ buscando desentrañar los dispositivos ideológicos que son incorporados por las personas(profesionales), al punto de llegar a identificarse con los intereses de los grupos dominantes que les someten a obediencia y servidumbre, condicionando las prácticas profesionales.

Desde la Terapia Ocupacional Crítica Latinoamericana, Pino et $\mathrm{al}^{43}$ hacen referencia al concepto de "descolonizar la ocupación humana":

Es el desplazamiento y desprendimiento hacia la condición pluriversatil de la ocupación; comprender la ocupación como expresión histórica diversa, como praxis en constante transformación, como potencial humano que va mutando a través de las tensiones y luchas sociales, a través de los cambios de paradigmas y el desarrollo de las fuerzas productivas, desarrolladas al interior de las comunidades. 
Desideologizar la ocupación en el consumo de SPA, es dejar de entenderla como una actividad negativa, sino como una actividad humana que se debe comprender desde la historia de las personas y las colectividades en sus relaciones políticas, sociales y culturales en su realidad territorial.

Entendemos, en consonancia con Dobles Oropeza ${ }^{44}$, que la desideologización es la potenciación de las virtudes populares, lo cual representa una validación del trabajo desde las comunidades y territorios. Dobles vincula a Baró y Dussell con el desarrollo de una ética de la Liberación, que al respecto refiere que desde América Latina se debe discernir sistemas de opresión y trabajar junto a las víctimas/personas que usan SPA, construyendo comunidades críticas para lograr transformaciones sistémicas.

\section{Conclusiones}

La concepción ideológica influye sobre los paradigmas científicos y estos condicionan las teorías (sistematización del conocimiento para su replicación), las cuales a su vez conforman los modelos, cuya diversidad conceptual depende de distintas concepciones de mundo y de las relaciones que los individuos establecen con la realidad, condicionando los estilos de las prácticas profesionales. Consideramos que el consumo de SPA, recibe como ninguna otra situación de salud, una serie de estigmas y estereotipos generados por el filtro moral de profesionales que se centran en los déficits. Estas concepciones legitimadas por paradigmas tradicionales, colocan a las personas que usan SPA como sujetos de censura y castigo por la sociedad, o como sujetos sin capacidades y habilidades para ser partícipes de su propio proceso de mejora de vida y bienestar; limitando la generación de estrategias basadas en evidencia, la diversificación de las metas de tratamiento y la oferta de servicios.

La práctica profesional relacionada con el consumo de SPA, sigue estando atravesada por ideologías de control social y no centrada en la persona y su contexto relacional, con esto buscamos estimular un compromiso reflexivo en las prácticas de Psicología y Terapia Ocupacional, partan desde los saberes territoriales de las personas y sus comunidades y no desde el sistema normativo, alejándonos de proyecciones individualistas, hedonistas, represivas y autoritarias ${ }^{44}$, transmitidas por el modelo de salud imperante.

Desde lo anterior, las nuevas perspectivas de atención tienen varios retos en la formación de profesionales de la salud con sentido crítico y visión integradora. Un primer reto es la ruptura con la visión clásica, al entender al sujeto de estudio como persona libre y autónoma, comprendiendo el uso de sustancias como una ocupación humana producto de la relación continua entre la persona y el entorno socio cultural. Desde esta mirada el uso de SPA como ocupación humana, en consecuencia, con Guajardo Córdoba ${ }^{45}$ no es un resultado de la mediación con el ambiente, es cultura, significado y relaciones históricas.

Otro reto fundamental es la ruptura de la noción de la abstinencia como única meta posible en el abordaje por el uso SPA. Miller ${ }^{27}$ indica que, en el pasado, el tratamiento contra la adicción se consideraba exitoso sólo si el cliente se volvía abstemio y no volvía a usar la sustancia después de ser dado de alta; una meta que quedó demostrada cuán difícil era de lograr. El enfoque se centraba casi por completo en hacer que el cliente dejase de usar la sustancia y comenzase a entender la naturaleza de su adicción. Hoy día, las metas del tratamiento incluyen una gama amplia de 
medidas biopsicosociales, como la reducción en el uso de sustancias, mejoramiento en la salud y funcionamiento psicosocial, mejoramiento en la estabilidad de empleo y reducción en la actividad criminal $^{27}$.

Otro reto que se colige del anterior, es que debemos considerar que la recuperación es de por sí multifacética, y que los logros conseguidos en miras a la recuperación, pueden aparecer en un aspecto de la vida del individuo, aunque no en otros ${ }^{31}$; lograr la meta de la abstinencia no necesariamente se traduce en un funcionamiento mejorado en la vida de las personas.

En consecuencia, con lo anterior, otra tarea emergente de los profesionales en salud con actitud desideologizante es visibilizar las contradicciones entre los enfoques, teorías y modelos, condicionados por la perspectiva ideológica dominante. Los profesionales podrían entender que el objetivo del acompañamiento terapéutico a personas que usan SPA, no es el énfasis en la droga, sino en las funcionalidades y significados de esta para la persona, donde lo importante es intentar que esta actividad humana no tome un valor único, en la gama de actividades significativas que pueden formar parte de su vida cotidiana, con o sin consumo.

Nuestro país no ha sido ajeno a esta transición epistemológica. La finalidad última de este artículo es crear conciencia en los agentes de servicios de salud, sobre la necesidad de desideologizar sus percepciones, prácticas, abordajes y acompañamientos, de los juicios de valor, estigmas y estereotipos provenientes de los modelos tradicionales moral, jurídico-punitivo, centrados en la enfermedad. De esta manera, poder trabajar realmente con las personas en su cotidianidad, sin juzgamientos ni valoración de sus elecciones o prácticas de vida, creyendo en sus capacidades, potencialidades y poder de cambio, según sus posibilidades, necesidades y realidades, en un contexto de constante interacción ambienteindividuo, ya que como refiere De Las Heras ${ }^{3}$ estas posturas de los grupos sociales o equipos de trabajo, se mantendrán como convicciones hasta que de alguna manera se genere un cambio en el conocimiento.

\section{Referencias bibliográficas}

1. Organización Mundial de la Salud. Marco sobre servicios de salud integrados y centrados en la persona. $69^{\circ}$ Asamblea Mundial de la Salud A/69/39 [Internet]. Ginebra: OMS; 2016. Disponible en: https://apps. who.int/gb/ebwha/pdf_files/WHA69/A69_39sp.pdf

2. Organización Mundial de la Salud. Seventieth World Health Assembly. Public health dimension of the world drug problem [Internet]. Ginebra: OMS; 2017. Disponible en: https://apps.who.int/gb/ebwha/pdf_files/ WHA70-REC1/A70_2017_REC1-en.pdf

3. De Las Heras G. Modelo de Ocupación Humana. Madrid: Síntesis; 2015.

4. Livingston JD, Boyd JE. Correlates and consequences of internalized stigma for people living with mental illness: A systematic review and metaanalysis. Social Sci Med. 2010;71(12):2150-2161. doi: 10.1016/j. socscimed.2010.09.030

5. Organización Mundial de la Salud. CDI-10- International Classification of Diseases and Related Health Problems. 10a ed. Ginebra: OMS; 2011.

6. Franco A. Tendencias y Teorías en Salud Pública. Rev Fac Nac Salud Pública [Internet]. 2006;24(2):119-130. Disponible 
en: http://www.scielo.org.co/pdf/rfnsp/v24n2/ v24n2a12.pdf

7. Mora Rodríguez A. Perspectivas filosóficas del hombre. San José, CR: EUNED; 1999.

8. Kuhn T. La Estructura de las Revoluciones Científicas. México: Fondo de Cultura Económica; 1971.

9. Nateras Domínguez A, Nateras Domínguez $\mathrm{O}$. El uso social de drogas: una mirada deconstruccionista. Iztalapa [Internet]. 1994;14(35):113-130. Disponible en: https://dialnet.unirioja.es/descarga/articulo/7086429.pdf

10. Kornblit AL, Camarotti AC, Di Leo PF. Prevención del consumo problemático de drogas, Modulo 1: La construcción social de la problemática de las drogas [Internet]. Buenos Aires: Ministerio de Educación (AR); 2010. Disponible en: http://files.unicef.org/ argentina/spanish/Edu_ModulosESI.pdf

11. Goffman E. Estigma, La identidad deteriorada. Buenos Aires: Amorrortu Editores; 1963

12. Mora M. La teoría de las representaciones sociales de Serge Moscovici. Athenea Digital. 2002;(2). doi: 10.5565/rev/athenead/v1n2.55

13. Althusser L. Ideología y aparatos ideológicos del Estado; 1969.

14. Ministerio de Salud (CR). Política Nacional de Salud Mental 2012-2021 [Internet]. San José: Ministerio de Salud; 2012. Disponible en: https://www.ministeriodesalud.go.cr/index.php/biblioteca-de-archivos/ sobre-el-ministerio/politcas-y-planes-en-salud/politicas-en-salud/1558-politica-nacio- nal-de-salud-mental/file

15. Ministerio de Salud (CR). Política del sector salud para el tratamiento de las personas con problemas derivados del consumo de alcohol, tabaco y otras drogas en Costa Rica [Internet]. San José: IAFA; 2012. Disponible en: https://www.iafa.go.cr/images/contenido/articulos/acerca-de-iafa/PoliticaTratamientoAdiccionesIAFA2012.pdf

16. Ministerio de Desarrollo Humano e Inclusión Social (CR). Política Nacional Atención Integral para Personas en Situación de Abandono y en Situación de Calle 2016-2026 [Internet]. San José: Ministerio de Desarrollo Humano e Inclusión Social; 2016. Disponible en: https://www.iafa.go.cr/images/contenido/ articulos/acerca-de-iafa/Documento $\% 20$ Poli\%C4\%9Btica\%20final\%203\%20de\%20 julio\%20(5-7-16).pdf

17. Instituto de Alcoholismo y Farmacodependencia (CR). Modelo de Reducción de Daños para el abordaje del fenómeno de drogas en Costa Rica [Internet]. San José: IAFA; 2017. Disponible en: https://www. iafa.go.cr/images/descargables/MODELO-DE-REDUCCION-DE-DAOS_2017.pdf

18. Instituto Costarricense sobre Drogas. Plan Nacional sobre Drogas, Legitimación de Capitales y Financiamiento al Terrorismo 2020-2024 [Internet]. San José: ICD; 2020. Disponible en: https://www.icd.go.cr/ portalicd/images/docs/icd/marco_estrategico/Informes_Cumplimiento/ENDDA-PNSD/ PNsD_2020-2024_arte.pdf

19. Ministerio de Salud (CR). Estrategia Nacional para el Abordaje Integral y la Reducción del Consumo de Sustancias Psicoactivas con Enfoque de Salud Pública (2016-2021). San José: Ministerio de Salud; 
2016. Disponible en: https://www.ministeriodesalud.go.cr/sobre_ministerio/directrices_ dm/DAJ_decreto_41386_estrategia_abordaje_sustancias_psicoactivas.pdf

20. Cersósimo Guzmán G. Los Estereotipos del Costarricense. San José: Editorial Universidad de Costa; 1993.

21. Instituto sobre Alcoholismo y Farmacodependencia (CR). V Encuesta Nacional sobre consumo de sustancias psicoactivas en población de Educación Secundaria 2018 [Internet]. San José: IAFA; 2019. Disponible en: https://www.iafa.go.cr/images/descargables/investigaciones/iafa-encuenstanacional-adolescentes-2019-web.pdf

22. Organización Mundial de la Salud. Global status report on alcohol and health 2018 [Internet]. Ginebra: OMS; 2018. Disponible en: https://apps.who.int/iris/bitstream/ handle/10665/274603/9789241565639-eng. pdf

23. Organización Mundial de la Salud. Estrategias para reducir el uso nocivo del alcohol: proyecto de estrategia mundial [Internet]. Ginebra: OMS; 2011. Disponible en: https://apps.who.int/gb/ebwha/pdf_files/ WHA63/A63_13-sp.pdf?ua=1

24. Nutt DJ, King LA, Phillips LD; Independent Scientific Committee on Drugs. Drug harms in the UK: a multicriteria decision analysis. Lancet. 2010;376(9752):155865. doi: 10.1016/S0140-6736(10)61462-6.

25. Amador G, Cortés E. Políticas de Drogas y Salud Pública, ¿Costa Rica ha hecho lo suficiente?. Perspectivas [Internet]. 2016;9. Disponible en: https://library.fes.de/ pdf-files/bueros/fesamcentral/12774.pdf

26. Miller W, Rollnick S. La Entrevista
Motivacional. España: Paidós Ibérica; 1999.

27. Miller W. Mejorando la Motivación para el Cambio en el Tratamiento de Abuso de Sustancias [Internet]. Maryland: Departamento de Salud y Servicios Humanos. Disponible en: https://web.vocespara.info/ comparte/2017_vcs/Drogodependencias_y_ adicciones/Mejorando_la_motivacion_para_ el_cambio_en_el_tratamiento_de_abuso_ de_sustancias.pdf

28. Narcóticos Anónimos. Narcóticos Anónimos (Libro Azul). California: Oficina de Servicios Mundiales de Narcóticos Anónimos; 1991.

29. Heim D. Addiction: Not just brain malfunction. Nature. 2014;507(7490):40. doi: 10.1038/507040e.

30. Miller W, Rollnick S. La Entrevista Motivacional. Ayudar a las personas a cambiar. 3a ed. España: Paidós Ibérica; 2015.

31. Tatarsky A. Psicoterapia de Reducción de Daños. Un nuevo tratamiento para problemas de drogas y alcohol [Internet]. New York: Rowman y Litlefield Publishers; 2002. Disponible en: https://static1. squarespace.com/static/58b08b6ae6f2e153c4932ea9/t/5eef54ac9f71275e5843bde4/1592743098686/Libro-Tatarsky_3_final. pdf

32. Schnake A. Los diálogos del cuerpo, un enfoque holístico de la salud y la enfermedad. Santiago de Chile: Cuatro Vientos; 1995.

33. Prochaska J, DiClemente C, Norcross J. Changing for Good: A Revolutionary Six-Stage Program for Overcoming Bad Habits and Moving Your Life Positively Forward. California: Harper Collins Publishers; 2007. 
34. Marlatt A, Gordon J. Relapse Prevention, Maintenance Strategies in the Treatment of Addictive Behaviors. New York: Guilford Press; 1985.

35. Marlatt A, Donovan D. Relapse Prevention, Maintenance Strategies in the Treatment of Addictive Behaviors. 2a ed. New York: Guilford Press; 2005.

36. Marlatt A, Larimer M, Witkiewitz K. Harm Reduction, Pragmatic Strategies for Managing High-Risk Behaviors. 2a ed. New York: Guilford Press; 2012.

37. Oficina de Naciones Unidas contra la Droga y el Delito. International Standards for the treatment of drug use disorders [Internet]. Ginebra: OMS; 2020. Disponible en: https:// apps.who.int/iris/rest/bitstreams/1273579/ retrieve

38. Moncho Cafelao. Martín Baró. Ignacio - Hacia una Psicología de la Liberación 1ra parte [video en Internet]. Youtube; 2013. Disponible en: https://www.youtube.com/ watch? $=7 w 4 i 2 n T 9 v$ Vo\&t $=601 \mathrm{~s}$

39. Martín Baró I. Del Alcohol a la Marihuana [Internet]. El Salvador: Estudios Centroamericanos; 1972. Disponible en: https://www.uca.edu.sv/coleccion-digital-IMB/ wp-content/uploads/2015/10/1972delalcoholalamarihuanaECA1972-27-283-225_242. pdf

40. De Las Heras C, LLerena V, Kielhofner G. Proceso de Remotivación: Intervención progresiva para personas que experimentan desafíos volitivos severos (Versión 2.0). College of Applied Health Sciences; 2019.

41. Cortés Amador E, Amighetti López D. Políticas de Drogas y Derechos Humanos:
Reformas en Costa Rica. Perspectivas [Internet]. 2014;4. Disponible en: https://library. fes.de/pdf-files/bueros/fesamcentral/10860. pdf

42. Barrero Cuellar E. Psicología de la Liberación: Del discurso encantador a la praxis liberadora. Aportes para la construcción de una psicología desde el sur [Internet]. Bogotá: Cátedra Libre; 2015. Disponible en: http://www.alfepsi.org/wp-content/ uploads/2015/07/PSICOLOGIA_DE_LA_LIBERACION.pdf

43. Pino J, Ulloa F. Perspectiva crítica desde Latinoamérica: hacia una desobediencia epistémica en terapia ocupacional contemporánea. Cad Ter Ocup UFSCar. 2016;24(2):421-427. doi: 10.4322/01044931.ctoARF0726

44. Dobles Oropeza I. Ignacio Martín Baró y la Psicología de la Liberación: un Desafío Vigente. En: González M (comp). Teorías Psicosociales. San José: Editorial Universidad de Costa Rica; 2010. 208.

45. Guajardo Córdoba A. Construcción de identidades, epistemes y prácticas en terapia ocupacional en América Latina. En: Simó S, Guajardo A, Correa F, Galheigo S, García S, editores. Terapias ocupacionales desde el Sur: derechos humanos, ciudadanía y participación. Santiago de Chile: USACH; 2016.41-62. 